\title{
Geographical mobility of general practitioners in rural Australia
}

\author{
GPs working \\ in small \\ communities
}

and those in a

rural location

for less than

3 years are

most at risk of

leaving rural

practice

Matthew R McGrail BSc(Hons), GradDiplT, PhD

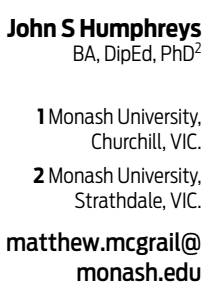

doi: 10.5694/mjal4.01375
$\mathrm{K}$ ey to improving the poorer health status that characterises people in rural areas is ensuring equitable access to appropriate health care..$^{1-3}$ However, this requires recruiting and retaining an adequate supply of appropriate health workers, which is known to be difficult in rural and remote areas. ${ }^{4,5}$ While considerable research has been conducted on the factors and barriers that facilitate and impede medical workforce supply in rural areas, there is a dearth of quantitative empirical evidence relating to the dynamics of general practitioner mobility patterns - specifically, which doctors move where, at what frequency, and why.

Understanding GP mobility is important because of its impact on workforce availability - both in the origin area (place from which the doctor moved) and the destination area. Considerable investment is made by governments into health programs specifically oriented towards improving the recruitment and retention of doctors in rural areas, with the goal of maximising movement into and minimising movement away from rural areas.

Despite a large body of social sciences literature on both inter- and intraregional migration, its applicability to the health workforce is not clear. Unfortunately, research literature focusing specifically on medical and health workforce mobility is scant. Internationally, sentinel works related to doctors include one 20-year national study in the United States on the volume and location of rural moves, although covariate analysis was not reported. ${ }^{6}$ Subsequent publications from the same dataset have focused on mobility in and out of areas of high need and between four major regions of the US. ${ }^{7,8}$ Similarly, a few Canadian studies have focused specifically on interregional (large distance) migration patterns of doctors without a focus on rural areas per se., ${ }^{9,10}$ Much of the extant health mobility literature has concentrated

\section{Abstract}

Objective: To describe the geographical mobility of general practitioners in Australia, both within rural areas and between rural and metropolitan areas.

Design and participants: Annual panel survey of GPs between 2008 and 2012.

Main outcome measures: Work location, categorised by a typology based on geographical location and community size; frequency of mobility (change of location category); and characteristics of those who moved.

Results: There were 3906 participants in 2008 (representative cohort, 19\% of Australia's GP workforce) and 3502, 3514, 3287 and 3361 in subsequent years. 1810 GPs participated in all 5 years, and 10900 origin-destination pairs were observed after removing GP registrars from the dataset. A total of 133 GPs moved from rural to metropolitan locations, 103 moved from metropolitan to rural locations, and 271 observed location changes were within rural areas. Annual location retention rates were $95 \%$ in regional centres, $90 \%$ in small rural towns and $82 \%$ in very remote areas. GPs in small towns of $<5000$ residents had the highest risk of leaving rural practice. Mobility rates were significantly higher for GPs who had worked in a location for under 3 years and those working as either contracted or salaried employees, and somewhat higher for international medical graduates. Younger age was a small predictor of increased mobility, while sex and family status had no association with mobility.

Conclusion: GPs working in small communities and those in a rural location for less than 3 years are most at risk of leaving rural practice.

on the international migration of doctors from developing to developed countries, ${ }^{11}$ focusing particularly on ethical issues, the impact of the loss of doctors on reduced access to health care in origin countries, and the roles of these international medical graduates (IMGs) in destination countries. ${ }^{12-14}$

Associations between mobility and covariates have rarely been quantified, $, 15,16$ with younger age being the dominant common factor linked with increased mobility. Much less has been written about the mobility of Australian doctors, ${ }^{17,18}$ and no specific mobility data have been published for the non-medical health workforce. The reasons for this lack of literature include limited access to data at a suitable geographical scale; lack of longitudinal studies from which to monitor doctors' movements; inherent difficulties of tracking individual doctors without linked datasets; and insufficient numbers of moves to generate valid and reliable results.
In an attempt to redress this paucity of evidence, we aimed to describe the geographical mobility of GPs in Australia both within rural areas and between rural and metropolitan areas. We describe where doctors are moving to and from, how many doctors are moving, and the characteristics of doctors who move. Such research helps to provide the basis for better understanding the role of push and pull factors behind why doctors move and what has influenced their decision to move. This in turn assists policymakers to design policies targeting medical workforce maldistribution in rural and remote areas.

\section{Methods}

We used data from the large Medicine in Australia: Balancing Employment and Life (MABEL) survey, conducted within the Centre for Research Excellence in Medical Workforce Dynamics. MABEL is Australia's national longitudinal survey of doctors, which collects similar data in annual waves from mostly the same 
panel of doctors (https://mabel.org. au). MABEL was approved by the University of Melbourne Faculty of Business and Economics Human Ethics Advisory Group (Reference 0709559) and the Monash University Standing Committee on Ethics in Research Involving Humans (Reference CF07/1102 - 2007000291).

\section{Study participants}

The first wave of the MABEL survey, in 2008, invited the participation of the entire medical workforce, and 3906 GPs (19\% of Australia's GP workforce) completed the initial survey. Subsequent annual waves of previous respondents saw a $70 \%-80 \%$ retention rate, including the annual addition of new GPs to the dataset and returning participants who missed at least 1 year. This study used data from waves 1-5 (2008 to 2012), comprising 3502 (wave 2), 3514 (wave 3), 3287 (wave 4) and 3361 (wave 5) responses. Detailed non-response bias was conducted for waves 1 and $2 .^{19,20}$ The most notable observable bias was a significant increase in the number of responses from doctors in remote areas, attributable to a financial incentive ( $\$ 100$ honorarium) to maximise participation of these GPws. GP registrars were excluded, because many do not have autonomy over their work location during their fellowship training.

\section{Locational measures}

Locational data were geocoded to a specific town or suburb. Each GP's self-reported work location $(<1 \%$ missing data) was used to calculate mobility, by comparing their location between each annual wave. Mobility was classified using the seven-category Modified Monash Model scale, ${ }^{21}$ which combines population size of settlements (<5000; 5000-15000; $>15000-50000$; and >50000) with the Australian Statistical Geography Standard - Remoteness Areas (ASGS-RA) classification, to define a geographical classification of most relevance to Australian GPs. ${ }^{22}$ Locational changes within the same rural town or within metropolitan areas were all classified as "no change".

\section{Statistical analysis}

Analysis was conducted in two distinct parts. First, all GP respondents were analysed using only their origin location and destination location, aggregated using the Modified Monash Model. GPs who participated in all five waves thus contributed four origin-destination pairs. Second, mobility outcomes were assessed for their association with additional key covariates of age $(<40,40-54$, $\geqslant 55$ years), sex, having a life partner, IMG status and location restrictions as part of their registration, business relationship within the practice, and length of stay in that location. For this analysis, mobility was categorised as no change (metropolitan), no change (rural), change from rural to metropolitan, change from metropolitan to rural and change within rural areas (where "rural" encompasses all six non-metropolitan categories, from regional to very remote). Annual "risk" of moving between rural and metropolitan locations was measured using total number of observed years. Panel (clustered) logit models were additionally used to measure the associations between these risk factors and either leaving rural areas (models 1 and 2) or leaving metropolitan areas (models 3 and 4). Length of stay was removed from models 2 and 4 because of its strong multicollinearity. All calculations were performed using StataSE 12 (StataCorp) with a $5 \%$ significance level.

\section{Results}

Between wave 1 and wave 5, a total of 5844 GPs completed at least one MABEL survey. Of these, 1810 GPs completed all five waves, providing 7240 mobility observations. A further 805 GPs missed one survey (2415 mobility observations), 786 completed three out of five waves (1572 observations) and 887 completed only two waves. Additionally, 1470 GPs completed only one MABEL survey, contributing no mobility data. In total, there were 12114 mobility observations, which decreased to 10900 after GP registrars were removed from the dataset.

Overall, fully trained GPs were observed to have a mobility rate of about 4.6\% (507/10900). In comparison, GP registrars had a mobility rate of $21 \%(253 / 1214)$.

Box 1 summarises the number of locational changes for the five waves (2008-2012). Cells along the main diagonal represent GPs who did not change their location between waves. This approximation of retention

1 Summary of origin-destination work location changes* for all non-registrar general practitioners (annual survey, 2008-2012)

\begin{tabular}{lccccccc} 
& \multicolumn{9}{c}{ Destination location, no. (\%) } & \multicolumn{1}{c}{ Total } \\
\cline { 2 - 7 } Origin location & Metropolitan & $\begin{array}{c}\text { Regional } \\
\text { centre }\end{array}$ & Large rural & $\begin{array}{c}\text { Medium } \\
\text { rural }\end{array}$ & Small rural & Remote & Very remote \\
$(n=10900)$
\end{tabular}

* There were an additional 51 work location changes observed within non-metropolitan areas where the location category was unchanged. 
within each category shows a decreasing rate as the degree of geographical rurality or remoteness increases. In cells to the right of the diagonal, the destination location is increasingly remote compared with the origin location (eg, large rural to remote), and cells to the left of the diagonal capture GPs who have moved to decreasingly remote locations (eg, small rural to medium rural). The first row captures all GPs (103) who moved from a metropolitan origin to a non-metropolitan destination during the five-wave period; $98.6 \%$ (7015) stayed within a metropolitan location. The first column of Box 1 captures all GPs who moved from a regional, rural or remote origin to a metropolitan destination (133). Just under half of all observed location changes were between non-metropolitan and metropolitan locations (236). There were 478 observations originating from a remote or very remote location, with 417 (87\%) GPs remaining within the same location in the next year, and most (45 [74\%]) of the 61 movers remaining within a non-metropolitan area.

Aggregate counts of GPs and the characteristics of the movers and stayers are summarised in Box 2. The observed risk (per observed year) of moving to a non-metropolitan area was 1 in 75 for metropolitan GPs. In contrast, the risk of losing nonmetropolitan GPs to metropolitan areas was 1 in 31 . Of the $271 \mathrm{GPs}$ who moved within non-metropolitan Australia, 77 moved to regional centres (population over 50000), but only 24 left regional centres for a smaller rural or remote location. A further 18 GPs moved from a rural to a remote location and 35 moved from remote practice to small or large rural locations.

Box 2 also shows the characteristics of GPs who moved compared with GPs who stayed in their original location. There was a small increased risk of moving for the youngest group of GPs, while sex and having a life partner had minimal association with increased mobility. IMGs had an increased risk of moving; even more so for the subgroup who were restricted in their location choice. GPs who were also principals of their practice were much less likely to move, while contract employees were

2 Characteristics of general practitioners who remain in or change their work location (annual survey, 2008-2012)

\begin{tabular}{|c|c|c|c|c|c|c|c|}
\hline \multirow[b]{2}{*}{$\begin{array}{l}\text { Origin location } \\
\text { characteristic }\end{array}$} & \multirow[b]{2}{*}{$\begin{array}{l}\text { No change: } \\
\text { metropolitan }\end{array}$} & \multirow[b]{2}{*}{$\begin{array}{l}\text { Metropolitan } \\
\text { to rural* }\end{array}$} & \multirow[b]{2}{*}{$\begin{array}{c}\text { Rural to } \\
\text { metropolitan }\end{array}$} & \multirow[b]{2}{*}{$\begin{array}{c}\text { No change: } \\
\text { rural }\end{array}$} & \multirow[b]{2}{*}{$\begin{array}{l}\text { Moved within } \\
\text { rural }\end{array}$} & \multicolumn{2}{|c|}{ Per-year "risk" } \\
\hline & & & & & & $\begin{array}{c}\text { Rural to } \\
\text { metropolitan }\end{array}$ & $\begin{array}{l}\text { Metropolitan } \\
\text { to rural }\end{array}$ \\
\hline Total observations & 7015 & 103 & 133 & 3378 & 271 & $3.20 \%(1$ in 31$)$ & $1.34 \%(1$ in 75$)$ \\
\hline \multicolumn{8}{|l|}{ Age group } \\
\hline$<40$ & $751(11 \%)$ & $16(16 \%)$ & $28(21 \%)$ & $335(10 \%)$ & $44(17 \%)$ & $6.3 \%(1$ in 16$)$ & $1.9 \%(1$ in 53$)$ \\
\hline 40-54 years & $3171(46 \%)$ & $41(40 \%)$ & $68(52 \%)$ & $1722(52 \%)$ & $136(52 \%)$ & $3.2 \%$ (1 in 31$)$ & $1.2 \%(1$ in 85$)$ \\
\hline $55+$ years & $3006(43 \%)$ & $45(44 \%)$ & $35(27 \%)$ & $1281(38 \%)$ & $84(32 \%)$ & $2.3 \%(1$ in 44$)$ & $1.4 \%(1$ in 73$)$ \\
\hline \multicolumn{8}{|c|}{ Sex and family circumstances } \\
\hline Male and partner & 3007 (45\%) & $40(42 \%)$ & $64(50 \%)$ & $1835(58 \%)$ & $136(54 \%)$ & $2.9 \%$ (1 in 35) & $1.2 \%(1$ in 83$)$ \\
\hline Female and partner & $2783(42 \%)$ & $38(40 \%)$ & $51(40 \%)$ & $1024(32 \%)$ & $87(35 \%)$ & $4.0 \%(1$ in 25$)$ & $1.3 \%$ (1 in 79) \\
\hline Male and no partner & $306(5 \%)$ & $8(8 \%)$ & $5(4 \%)$ & $141(4 \%)$ & $17(7 \%)$ & $2.7 \%$ ( 1 in 37$)$ & $2.4 \%(1$ in 42$)$ \\
\hline Female and no partner & $517(8 \%)$ & $10(10 \%)$ & $8(6 \%)$ & $180(6 \%)$ & $10(4 \%)$ & $3.8 \%(1$ in 27$)$ & $1.8 \%(1$ in 57$)$ \\
\hline \multicolumn{8}{|c|}{ Training location and place restriction ${ }^{\dagger}$} \\
\hline Local, unrestricted & $5282(80 \%)$ & $66(71 \%)$ & $70(54 \%)$ & $2226(70 \%)$ & $145(56 \%)$ & $2.6 \%(1$ in 38$)$ & $1.1 \%$ (1 in 87) \\
\hline IMG, restricted & $295(4 \%)$ & $11(12 \%)$ & $35(27 \%)$ & $416(13 \%)$ & $60(23 \%)$ & $6.2 \%(1 \mathrm{in} 16)$ & $3.3 \%(1$ in 30$)$ \\
\hline IMG, unrestricted & $1022(15 \%)$ & $16(17 \%)$ & $24(19 \%)$ & $519(16 \%)$ & $54(21 \%)$ & $3.6 \%(1$ in 28$)$ & $1.4 \%(1$ in 72$)$ \\
\hline \multicolumn{8}{|l|}{ Business relationship } \\
\hline Principal, partner & $2119(33 \%)$ & $15(16 \%)$ & $16(13 \%)$ & $1211(39 \%)$ & $38(18 \%)$ & $1.2 \%(1$ in 87$)$ & $0.6 \%(1 \mathrm{in} \mathrm{155)}$ \\
\hline Associate & $664(10 \%)$ & $11(12 \%)$ & $13(11 \%)$ & $469(15 \%)$ & $20(9 \%)$ & $2.4 \%(1$ in 41$)$ & $1.5 \%(1$ in 67$)$ \\
\hline Salaried employee & $371(6 \%)$ & $13(14 \%)$ & $15(13 \%)$ & $307(10 \%)$ & $36(17 \%)$ & $3.9 \%(1$ in 26$)$ & $3.1 \%(1$ in 32$)$ \\
\hline Contract employee & $3261(51 \%)$ & $52(57 \%)$ & $76(63 \%)$ & $1086(35 \%)$ & $119(56 \%)$ & $5.4 \%(1$ in 19$)$ & $1.5 \%(1$ in 68$)$ \\
\hline \multicolumn{8}{|c|}{ Length of stay in origin location } \\
\hline$\leqslant 1$ year & $533(8 \%)$ & $24(26 \%)$ & $40(33 \%)$ & $416(13 \%)$ & $68(29 \%)$ & $6.8 \%(1$ in 15$)$ & $3.8 \%(1$ in 26$)$ \\
\hline $2-3$ years & $722(11 \%)$ & $18(19 \%)$ & $32(26 \%)$ & $414(13 \%)$ & $50(21 \%)$ & $5.8 \%(1$ in 17$)$ & $2.2 \%(1$ in 45$)$ \\
\hline $4-6$ years & $894(14 \%)$ & $14(15 \%)$ & $11(9 \%)$ & $406(13 \%)$ & $47(20 \%)$ & $2.2 \%(1$ in 46$)$ & $1.4 \%(1$ in 70$)$ \\
\hline $7-10$ years & $917(14 \%)$ & $8(9 \%)$ & $12(10 \%)$ & $396(13 \%)$ & $25(11 \%)$ & $2.6 \%(1$ in 39$)$ & $0.8 \%(1$ in 125$)$ \\
\hline $11+$ years & 3491 (53\%) & $30(32 \%)$ & $26(21 \%)$ & $1521(48 \%)$ & $48(20 \%)$ & $1.5 \%(1$ in 67$)$ & $0.8 \%(1$ in 125$)$ \\
\hline
\end{tabular}

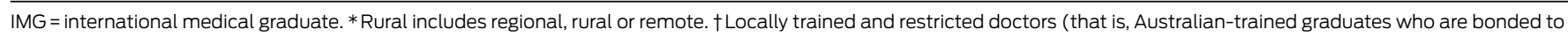
initially work in a rural area) have been removed from this analysis because there were very few observations in this group. 
highly mobile away from regional, rural and remote areas.

Box 3 shows the association between observed significant location changes and GP characteristics, with two binary outcomes tested (leaving rural and leaving metropolitan practice). Younger rural GPs were significantly more likely to leave rural practice than older rural GPs. There were no other significant associations between GP mobility and age or between GP mobility and sex and family status. The risk of moving to a metropolitan area was 2.5 to three times higher for rural GPs in their first 3 years in a location than for those who had been in a location for 4 years or more. Both contract employees and salaried employees were highly likely to leave rural practice, while salaried employees were most likely to leave metropolitan areas. Compared with GPs in regional centres, those in small and medium rural towns were significantly more likely to leave rural practice, while GPs in very remote areas had a lower risk of moving to a metropolitan area. The omission of length of stay strengthened the mobility odds ratio of all employment types, compared with practice principal or partners, and the association between small population size and an increased risk of turnover in rural areas. Additionally, IMGs restricted in their practice location had a higher risk of moving than Australian-trained, unrestricted GPs.

\section{Discussion}

This study provides the first national evidence of rural GP mobility over an extended period. Moreover, we investigated whether individual- and practice-level covariates were associated with the propensity to move. We used the seven-category Modified Monash Model to show which groups of GPs exhibit the highest mobility and are most at risk of leaving rural practice, or most likely to leave metropolitan areas for rural practice.

GPs in small rural towns and remote areas had higher mobility rates. While remote and very remote GPs had the highest mobility rate, this group was not significantly at increased risk of leaving non-metropolitan practice completely. Rural GPs practising in small towns (less than 5000 residents) and in medium-sized towns (up to 15000 residents) were most at risk of moving to metropolitan areas. These results further support the need for policies to better target GPs in small rural communities and differentiate them from GPs in large regional centres. ${ }^{21-23}$

GPs most at risk of moving, both from and to rural areas, are those who have only been in their current location for up to 3 years, similar to recent findings in rural New South Wales. ${ }^{24}$

3 Panel logit models of general practitioners who move between metropolitan and rural work locations (annual survey, 2008-2012)

\begin{tabular}{|c|c|c|c|c|}
\hline Origin location characteristic & $\begin{array}{l}\text { Leaving rural,* } \\
\text { model } 1^{\dagger}(\text { OR }[95 \% \\
\mathrm{CI}])\end{array}$ & $\begin{array}{l}\text { Leaving rural, model } 2^{\dagger} \\
(\text { OR }[95 \% \mathrm{CI}])\end{array}$ & $\begin{array}{c}\text { Leaving metropolitan, } \\
\text { model } 3^{\ddagger} \\
(\text { OR }[95 \% \mathrm{CI}])\end{array}$ & $\begin{array}{c}\text { Leaving metropolitan, } \\
\text { model } 4^{\ddagger} \\
(\text { OR }[95 \% \mathrm{CI}])\end{array}$ \\
\hline \multicolumn{5}{|c|}{ Age group (reference: $55+$ years) } \\
\hline$<40$ years & $2.06(0.79-5.34)$ & $2.85(1.09-7.43) * *$ & $0.62(0.30-1.27)$ & $1.11(0.57-2.14)$ \\
\hline $40-54$ years & $1.62(0.75-3.48)$ & $1.68(0.76-3.72)$ & $0.61(0.35-1.04)$ & $0.76(0.45-1.26)$ \\
\hline \multicolumn{5}{|c|}{ Sex and family status (reference: male and partner) } \\
\hline Female and partner & $1.07(0.54-2.10)$ & $1.13(0.56-2.27)$ & $0.97(0.57-1.63)$ & $0.90(0.54-1.50)$ \\
\hline Male and no partner & $1.35(0.35-5.27)$ & $1.51(0.37-6.14)$ & $1.32(0.50-3.46)$ & $1.56(0.64-3.79)$ \\
\hline Female and no partner & $0.62(0.15-2.47)$ & $1.07(0.29-3.91)$ & $1.26(0.56-2.80)$ & $1.20(0.54-2.65)$ \\
\hline \multicolumn{5}{|c|}{ Training location and place restriction (reference: Australia, unrestricted) } \\
\hline International, restricted & $0.91(0.41-2.05)$ & $1.57(0.73-3.36)$ & $1.59(0.74-3.41)$ & $2.65(1.29-5.43)^{\dagger \dagger}$ \\
\hline International, unrestricted & $1.39(0.58-3.32)$ & $1.92(0.79-4.66)$ & $1.02(0.54-1.92)$ & $1.33(0.73-2.41)$ \\
\hline \multicolumn{5}{|c|}{ Business relationship (reference: principal, partner) } \\
\hline Associate & $1.18(0.39-3.61)$ & $1.76(0.58-5.40)$ & $1.83(0.76-4.37)$ & $2.43(1.05-5.60) * *$ \\
\hline Salaried employee & $3.89(1.15-13.16) * *$ & $7.23(1.94-26.89)^{\dagger \dagger}$ & $3.22(1.33-7.82)^{\dagger \dagger}$ & $4.94(2.14-11.40)^{\dagger \dagger}$ \\
\hline Contract employee & $5.18(1.98-13.56)^{\dagger \dagger}$ & $8.38(2.72-25.78)^{\dagger \dagger}$ & $1.47(0.74-2.92)$ & $2.20(1.16-4.19) * *$ \\
\hline \multicolumn{5}{|c|}{ Length of stay in origin location (reference: $11+$ years) ${ }^{\natural}$} \\
\hline$\leqslant 1$ year & $3.40(1.34-8.62)^{\dagger \dagger}$ & - & $4.46(2.11-9.42)^{\dagger \dagger}$ & - \\
\hline $2-3$ years & $3.73(1.41-9.85)^{\dagger \dagger}$ & - & $2.63(1.25-5.53) * *$ & - \\
\hline $4-6$ years & $1.03(0.36-2.94)$ & - & $2.16(1.04-4.48) * *$ & - \\
\hline $7-10$ years & $0.86(0.30-2.48)$ & - & $1.24(0.54-2.85)$ & - \\
\hline \multicolumn{5}{|c|}{ Modified Monash rural scale (reference: regional centre) } \\
\hline Large rural & $2.25(0.78-6.50)$ & $2.68(0.88-8.20)$ & & \\
\hline Medium rural & $3.04(1.02-9.09) * *$ & $3.46(1.09-10.99)^{* *}$ & & \\
\hline Small rural & $3.96(1.41-11.14)^{\dagger \dagger}$ & $5.03(1.61-15.78)^{\dagger \dagger}$ & & \\
\hline Remote & $1.22(0.34-4.34)$ & $1.27(0.34-4.81)$ & & \\
\hline Very remote & $0.20(0.01-3.03)$ & $0.23(0.01-4.17)$ & & \\
\hline
\end{tabular}


That is, once a GP has been settled in either a rural or metropolitan location for at least 3 years they are less likely to move.

Additionally, younger GPs (under 40 years) and those working as either salaried or contract employees are more likely to be mobile. Sex and family status were not associated with mobility.

When more data become available, we plan to investigate whether there is any association between mobility and GPs' satisfaction with the schools that are available for their dependents.

Unrestricted rural IMGs had a slightly but non-significantly increased risk of leaving rural practice compared with locally trained unrestricted GPs. Further investigation of the strength of association between mobility and changed restriction (overseas trained) or bonding (Australian trained) status is also planned.

Our study was strengthened by the removal of GP registrars. GP registrars frequently have minimal control over their training locations, and so their moves are not equivalent to observed moves of GPs who have independently chosen to practise in a specific location. GP registrars are highly mobile, both between metropolitan and rural areas as well as between different rural areas. In this study, GP registrars were observed to have a mobility rate about five times higher than the annual mobility rate for fully trained GPs.

The main limitation of this study was our use of a self-selected cohort. Annually, the MABEL survey includes about $16 \%-19 \%$ of all Australian GPs, with $75 \%-80 \%$ of participants returning each year (potential selection or attrition bias). Despite having five annual waves of data, the number of GPs observed changing work location was still relatively small. In total, only 236 GPs were observed moving in either direction between rural and metropolitan areas. Larger numbers of moves are observable using more complete datasets like the Australian Health Practitioner Regulation Agency dataset, ${ }^{18}$ but this approach only provides very limited covariate information and includes large bias from the highly mobile GP registrar subgroup. The true mobility rate of rural-urban relocations for Australia's GP population may be different to the $3.2 \%$ (rural origin) and $1.3 \%$ (urban origin) observed annual rates in our study. More observed moves and sophisticated panel data analysis will be possible as additional MABEL data become available.

Increasing workforce supply and maintaining the existing rural medical workforce remains a key health issue in improving rural health in Australia. For several decades now, the Australian Government has made considerable investment in training more medical graduates, exposing these new doctors to more rural experience during their training, and increasing GP fellowships, rural bonded scholarships and rural retention payments. ${ }^{25}$ Nonetheless, little longitudinal evidence exists on how long to expect GPs to remain in different locations, what locations GPs move from and to, and personal and organisational factors associated with mobility. Most existing evidence comprises only cross-sectional data on retrospectively identified factors and prospective intention.

Using the best available GP data, this study helps to understand who is most likely to move each year, how often moves occur and where they might move to and from. In particular, these results both highlight and quantify the strong association between mobility propensity and increasing rurality and remoteness of practice locations. Such evidence is useful in guiding more effective targeting of rural health policies and workforce planning and incentives.

Acknowledgements: We used data from the Medicine in Australia: Balancing Employment and Life (MABEL) longitudinal survey of doctors conducted by the University of Melbourne and Monash University. Funding for MABEL comes from the National Health and Medical Research Council (Health Services Research Grant, 2008-2011; and Centre for Research Excellence in Medical Workforce Dynamics, 2012-2016) with additional support from the Department of Health (in 2008). We especially thank the doctors who gave their valuable time to participate in the MABEL survey.

Competing interests: No relevant disclosures. Received 25 Sep 2014, accepted 23 Apr 2015 .

References are available online at www.mja.com.au. 
1 World Health Organization. Increasing access to health workers in remote and rural areas through improved retention: global policy recommendations. Geneva: WHO, 2010. http://www.searo. who.int/nepal/mediacentre/2010_ increasing_access_to_health_workers_ in_remote_and_rural_areas.pdf (accessed Jun 2015).

2 Australian Institute of Health and Welfare. Rural, regional and remote health: indicators of health status and determinants of health. Canberra: AlHW, 2008. (Rural Health Series No. 9; AlHW Cat. No. PHE 97.) http:// www.aihw.gov.au/WorkArea/ DownloadAsset.aspx?id=6442459834 (accessed Jun 2015).

3 Humphreys JS, Solarsh G. At-risk populations: rural. In: Heggenhougen HK, Quah SR, editors. The international encyclopedia of public health. London: Academic Press, 2008.

4 Wakerman J, Humphreys JS. Sustainable workforce and sustainable health systems for rural and remote Australia. Med J Aust 2013; 199 (Suppl 5): 14-17.

5 Viscomi M, Larkins S, Sen Gupta T. Recruitment and retention of general practitioners in rural Canada and Australia a review of the literature. Can J Rural Med 2013; 18: 13-23.

6 Ricketts TC, Randolph R. Urban-rural flows of physicians. J Rural Health 2007; 23: 277-285.

7 Ricketts TC, Randolph R. The diffusion of physicians. Health Aff (Millwood) 2008; 27: 1409-1415.

8 Vanasse A, Ricketts TC, Courteau J, et al. Long term regional migration patterns of physicians over the course of their active practice careers. Rural Remote Health 2007; 7: 812.

9 McDonald JT, Worswick C. The migration decisions of physicians in Canada: the roles of immigrant status and spousal characteristics. Soc Sci Med 2012; 75: 1581-1588.

10 Rajbhandary S, Basu K. Interprovincial migration of physicians in Canada: Where are they moving and why? Health Policy 2006; 79: 265-273.
1 Dussault G, Franceschini MC. Not enough there, too many here: understanding geographical imbalances in the distribution of the health workforce. Hum Resour Health 2006; 4: 12.

12 Bourgeault IL, Baumann A. Ethical recruitment and integration of internationally educated health professionals in Canada. 13th International Health Workforce Collaborative; 2011 Oct 24-26; Brisbane, Australia; Royal College of Physicians and Surgeons of Canada, 2014. http:// rcpsc.medical.org/publicpolicy/imwc/ conferencel3.php (accessed Jun 2015).

13 Hawthorne L. Health workforce migration - Australia. 13th International Health Workforce Collaborative; 2011 Oct 24-26; Brisbane, Australia; Royal College of Physicians and Surgeons of Canada, 2014. http://rcpsc.medical. org/publicpolicy/imwc/conferencel3. php (accessed Jun 2015).

14 van Zanten M, Boulet JR, Norcini JJ. Ethical integration of internationally educated health professionals: ethical and regulatory contexts. 13th International Health Workforce Collaborative; 2011 Oct 24-26; Brisbane, Australia; Royal College of Physicians and Surgeons of Canada, 2014. http:// rcpsc.medical.org/publicpolicy/imwc/ conferencel3.php (accessed Jun 2015).

15 Inoue K, Matsumoto M, Toyokawa S, et al. Transition of physician distribution (1980-2002) in Japan and factors predicting future rural practice. Rural Remote Health 2009; 9: 1070.

16 Ricketts TC. The migration of physicians and the local supply of practitioners: a five-year comparison. Acad Med 2013; 88: 1913-1918.

17 Adhikari M, Calcino G, Dickinson J. Geographical mobility of general practitioners. Proceedings of the 2 nd National Rural Health Conference; 1993 Feb 12-14; Armidale, NSW, Australia; National Rural Health Alliance. http:// ruralhealth.org.au/PAPERS/2_MADIK. pdf (accessed Jun 2015).

18 Mazumdar S, McRae I. Doctors on the move: estimating geographic patterns of general practitioner (GP) mobility in Australia using a large registration database. 2014 Primary Health Care Research Conference; 2014 Jul 23-25; Canberra, Australia.

19 Joyce C, Scott A, Jeon S, et al. The "Medicine in Australia: Balancing Employment and Life (MABEL)" longitudinal survey - Protocol and baseline data for a prospective cohort study of Australian doctors' workforce participation. BMC Health Serv Res 2010; 10: 50.

20 Yan W, Cheng TC, Scott A, et al. Medicine in Australia: balancing employment and life (MABEL). Aust Econ Rev 2011; 44: 102-112.

21 Mason J (Chair). Review of Australian government health workforce programs ("Mason review"). Canberra: Department of Health and Ageing, 2013. https://www.health.gov. au/internet/main/publishing.nsf/ Content/D26858F4B68834EACA25 7BF0001A8DDC/\$File/Review\%20 of $\% 20$ Health\%20Workforce $\% 20$ programs.pdf (accessed Jun 2015).

22 Humphreys JS, McGrail MR, Joyce CM, et al. Who should receive recruitment and retention incentives? Improved targeting of rural doctors using medical workforce data. Aust J Rural Health 2012; 20: 3-10.

23 McGrail MR, Humphreys JS. Geographical classifications to guide rural health policy in Australia. Aust New Zealand Health Policy 2009; 6: 28.

24 Russell D, Humphreys JS, McGrail MR, et al. The value of survival analyses for evidence-based rural medical workforce planning. Hum Resour Health 2013; 11: 65.

25 Russell LM. Primary care and general practice in Australia 1990-2012: a chronology of federal government strategies, policies, programs and funding. Canberra: Australian Primary Health Care Research Institute, 2013. http://aphcri.anu.edu.au/files/ primary_care_and_general_practice_ update_may_2013.pdf (accessed Jun 2015). 